\title{
Injective mappings in $\mathbb{R}^{\mathbb{R}}$ and lineability*
}

\author{
P. Jiménez-Rodríguez S. Maghsoudi G.A. Muñoz-Fernández \\ J.B. Seoane-Sepúlveda
}

\begin{abstract}
It is known that there is not a two dimensional linear space in $\mathbb{R}^{\mathbb{R}}$ every non-zero element of which is an injective function. Here, we generalize this result to arbitrarily large dimensions. We also study the convolution of nondifferentiable functions which gives, as a result, a differentiable function. In this latter case, we are able to show the existence of linear spaces of the largest possible dimension formed by functions enjoying the previous property. By doing this we provide both positive and negative results to the recent field of lineability. Some open questions are also provided.
\end{abstract}

\section{Introduction and preliminaries}

In [12] W. Goldbloom Bloch gives several examples of open mappings between Euclidean spaces which are discontinuous. One of the first examples of functions of this kind was proposed by Lebesgue, and is given as follows: let $\Delta$ be the Cantor set and write $\mathbb{R} \backslash \Delta$ as a disjoint union of open sets $\left\{I_{k}\right\}$. For every $k$, let $f_{k}: I_{k} \rightarrow \mathbb{R}$ be a bijection. Define then

$$
L(x)= \begin{cases}x & \text { if } x \in \Delta, \\ f_{k}(x) & \text { if } x \in I_{k}\end{cases}
$$

As Goldbloom Bloch states, all the examples for an open discontinuous function require discontinuity at an infinite number of points. To give an example of a

\footnotetext{
*Supported by the Spanish Ministry of Science and Innovation, Grant MTM2015-65825-P.

Received by the editors in May 2016 - In revised form in June 2016.

Communicated by F. Bastin.

2010 Mathematics Subject Classification : Primary 28A20; Secondary 15A03, 26 A24.

Key words and phrases : lineability, open mapping, injective function, special functions.
} 
discontinuous open mapping which is continuous except at only a finite number of points, the author proposes

$$
f(z)= \begin{cases}0 & \text { if } z=0, \\ e^{1 / z} & \text { if } z \neq 0,\end{cases}
$$

defined over $\mathbb{C}$. This is an open mapping which is discontinuous only at 0 . Using the usual identification $\mathbb{C} \leftrightarrow \mathbb{R}^{2}$ we obtain an open mapping $f: \mathbb{R}^{2} \rightarrow \mathbb{R}^{2}$ which is discontinuous only at $(0,0)$.

In the following, we will study the extent to which the number of points of discontinuity is important when trying to find algebraic structure in sets of discontinuous open mapping. Recall that in the beginning of the $21^{\text {st }}$ century, the definitions that formalize the idea of "consider large algebraic structures inside of sets" were coined. To be more precise, we have the following:

Definition 1.1. $[3,4,7,10,11]$ Let $M$ be a subset of a topological vector space $X$ and $\mu$ and $v$ two cardinal numbers.

1. We say that $M$ is $\mu$-lineable if there exists a vector space $V \subseteq M \cup\{0\}$ of dimension $\mu$.

2. We say that $M$ is $\mu$-spaceable if there exists a closed vector space $V \subseteq M \cup\{0\}$ of dimension $\mu$. We say that $M$ is $(\mu, v)$-algebrable if there exists an algebra $V \subseteq M \cup\{0\}$ of dimension $\mu$ such that the cardinality of a minimal system of generators is $v$.

In the second half of the 19th century, the first examples for what we shall refer as (in mathematical folklore) pathological properties rose interest among mathematicians, and in 1875 Paul du Bois-Reymond published the proof for the existence of a continuous nowhere differentiable function. To express the shock that function provoked in most of mathematicians of that time, du Bois wrote: "It appears to me that the metaphysics of Weierstrass's function still hides many riddles and I cannot help thinking that entering deeper into the matter will finally lead us to the limits of our intellect."

The study of lineability (and other properties of subsets of topological vector spaces, together with the type of algebraic structure to be considered) tries to generalize the existence of those elements fulfilling pathological properties through finding large algebraic structures of such examples. Up to now, a great number of cases have been studied (giving even optimal results, talking about maximal dimension or cardinality). The monographs $[2,6]$ provide the state of the art of this topic.

In section 2, we will study two properties of functions: openness and, in relation with it, injectivity. It is interesting to stress that, even though most of the results that concerns lineability consist in finding explicitly structures of infinite dimension, we were able to prove that in most of this section that we can not go beyond finite dimension. One of the earliest results in this negative approach was the following: 
Theorem 1.2. $[1,9,16]$ The set of injective functions from $\mathbb{R}$ to $\mathbb{R}$ is not 2-lineable.

We will investigate the extent to which this theorem holds when seen in higher dimensions. We will use a corollary to the proof of the theorem, according to which, given $f, g$ injective functions and $x \neq y$, it is possible to construct a linear combination that attains the same value at $x$ and $y$.

In the last section of this article, we will complete one problem that was presented in [13], where the authors studied smoothing properties of the Volterra convolution. Even though the definition slightly differs from the usual definition of convolution, this operator still takes the 'best properties' of the functions involved. Thus, for example, the Volterra convolution of an $L_{1}$-function with a differentiable function having bounded derivative is still differentiable. Also, the Volterra convolution of two $L_{2}-$ functions gives a continuous function. Specifically, we have the following definition:

Definition 1.3. Let $f_{1}, f_{2}$ be two $L_{1}^{l o c}(\mathbb{R})$-functions. We define its Volterra convolution as

$$
f_{1} *_{V} f_{2}(x)=\int_{0}^{x} f_{1}(\zeta) f_{2}(x-\zeta) d \zeta .
$$

Observe that in the previous definition it is enough to consider locally $L_{1}$ functions, namely $L_{1}^{\text {loc }}(\mathbb{R})$.

The study of lineability or spaceability in relation to the smoothness of functions has been deeply carried out, and for example, in 1999, V.P. Font, V.I. Gurariy and M.I. Kadets proved the existence of a closed vector space of dimension $\mathfrak{c}$ all of whose elements except zero are continuous nowhere differentiable functions ([8]).

In [14], the authors were able to show that the set $\left\{W_{a}: \frac{7}{9}<a<1\right\}$ is a linearly independent set which spans a vector space of such functions, where

$$
W_{a}(x)=\sum_{k \geq 0} a^{k} \cos \left(9^{k} x\right)
$$

is the well-known Weierstrass' Monster, and in [13], the authors proved that in fact any non-trivial linear combination of elements of this set gives a function whose convolution with itself is nowhere differentiable. With this result, the authors of [13] showed that the set of continuous nowhere differentiable functions whose convolution with themselves gives a nowhere differentiable function is $\mathfrak{c}$-lineable.

One might think that the property of nowhere differentiability would indeed imply the nowhere differentiability of the Volterra convolution. However, in [13] an example is given of a continuous nowhere differentiable function whose convolution with itself is everywhere differentiable. In section 3 we complement this last result by giving an independent set of cardinality $\mathfrak{c}$ of nowhere differentiable functions such that, convolving two elements from the spanned vector space, we obtain a differentiable function. 


\section{Open, Continuous, and Injective mappings}

As stated in the preliminaries, we will first separate the situation where the considered functions are discontinuous at an uncountable set of points or they are continuous except a finite number of points. For the first case, just remark that $\mathcal{E S}(\mathbb{R}):=\{f: \mathbb{R} \rightarrow \mathbb{R}: f(I)=\mathbb{R}$ for every interval $I\}$ is a subset of the uncountably discontinuous open functions. We can then refer to [4], where it is proved that $\mathcal{E} \mathcal{S}(\mathbb{R})$ is $2^{\mathfrak{c}}$-lineable.

If we examine now the case where the open mappings are continuous except from a finite set, we have the following:

Theorem 2.1. The set of open mappings from $\mathbb{R}$ to $\mathbb{R}$ which are discontinuous at only a finite set of points is not 2-lineable.

Before giving the proof of this first result, we state a lemma that will be crucial for our purposes:

Lemma 2.2. A continuous open mapping $f: \mathbb{R} \rightarrow \mathbb{R}$ is injective.

Proof of theorem 2.1. Assume $f_{1}$ and $f_{2}$ are two open mappings, so that $f_{1}$ is continuous on $\mathbb{R} \backslash\left\{x_{1}, \ldots, x_{k}\right\}$ and $f_{2}$ is continuous on $\mathbb{R} \backslash\left\{y_{1}, \ldots, y_{l}\right\}$. Let us find $(a, b) \subseteq \mathbb{R}$ so that $f_{1}$ and $f_{2}$ are continuous on $(a, b)$.

We can choose then $a_{1}, a_{2} \in \mathbb{R} \backslash\{0\}$ so that $a_{1} f_{1}+a_{2} f_{2}$ is a nonzero noninjective continuous function on $(a, b)$. Use then Lemma 2.2 to show that the restriction of $a_{1} f_{1}+a_{2} f_{2}$ to $(a, b)$ is not open (and hence $a_{1} f_{1}+a_{2} f_{2}$ can not be open as a function on $\mathbb{R})$.

Remark 2.3. An analogous proof also works if we study the functions that are continuous except on a discrete set, a subset of a closed set with finite Lebesgue measure or a bounded set.

The proof of Theorem 2.1 relies deeply in the fact that the set of injective functions defined from $\mathbb{R}$ to $\mathbb{R}$ is not 2-lineable (see Theorem 1.2 of Section 1). In the following result we will generalize Theorem 1.2 to arbitrary dimensions:

Theorem 2.4. Let $n$ and $m$ be arbitrary. Then, the set $\left\{f: \mathbb{R}^{n} \rightarrow \mathbb{R}^{m}: f\right.$ is injective $\}$ is $m$-lineable, but not $m+1$-lineable.

Proof. Let $\Phi: \mathbb{R}^{n} \rightarrow \mathbb{R}$ be a bijection and consider the functions

$$
f_{i}=(\overbrace{e^{\Phi(x)}, \ldots, e^{\Phi(x)}}^{i}, \overbrace{0, \ldots, 0}^{m-i}) .
$$

Then, $\left\{f_{i}: 1 \leq i \leq m\right\}$ is a linearly independent set, any nontrivial linear combination of whose elements gives an injective function. Indeed, let

$$
f(x)=\sum_{i=1}^{m} a_{i} f_{i}(x)
$$


and assume $f(x)=f(y)$. Let $i_{0}=\max \left\{1 \leq i \leq m: a_{i} \neq 0\right\}$. Then, we can write

$$
f(x)=\left(\sum_{i=1}^{i_{0}} a_{i} e^{\Phi(x)}, \sum_{i=2}^{i_{0}} a_{i} e^{\Phi(x)}, \ldots, a_{i_{0}} e^{\Phi(x)}, 0, \ldots, 0\right),
$$

so $f(x)=f(y)$ yields $e^{\Phi(x)}=e^{\Phi(y)}$ and the first part of the proof follows.

Let now $g_{1}, \ldots, g_{m+1}: \mathbb{R}^{n} \rightarrow \mathbb{R}^{m}$ be functions and $x \neq y \in \mathbb{R}^{n}$. Set, for $1 \leq i \leq m+1, g_{i}(z)=\left(g_{i}^{(1)}(z), \ldots, g_{i}^{(m)}(z)\right)$ and define, for $1 \leq k \leq m$,

$$
u_{k}=\left(g_{1}^{(k)}(x)-g_{1}^{(k)}(y), \ldots, g_{m+1}^{(k)}(x)-g_{m+1}^{(k)}(y)\right) \in \mathbb{R}^{m+1} .
$$

Then, $\operatorname{dim}\left(u_{1}^{\perp} \cap \ldots \cap u_{m}^{\perp}\right) \geq 1$, so there exists $b \in \mathbb{R}^{m+1} \backslash\{0\}$ such that $\left\langle b, u_{k}\right\rangle=0$ for every $1 \leq k \leq m$.

If we consider the function $F=b_{1} g_{1}+\ldots+b_{m+1} g_{m+1}$, we obtain that $F$ cannot be injective. Indeed,

$$
\begin{aligned}
F^{(k)}(x) & =b_{1} g_{1}^{(k)}(x)+\ldots b_{m+1} g_{m+1}^{(k)}(x) \\
& =\left\langle b, u_{k}\right\rangle+b_{1} g_{1}^{(k)}(y)+\ldots b_{m+1} g_{m+1}^{(k)}(y)=F^{(k)}(y) .
\end{aligned}
$$

Related to this last result, we observe that the functions constructed in the proof are in general discontinuous (since we cannot have a continuous injective function $\Phi: \mathbb{R}^{n} \rightarrow \mathbb{R}$ for $n \geq 2$ ). We can wonder if we can have a similar lineability result for continuous injective functions. Unfortunately, we have not been able to prove lineability for all dimensions, but we can at least give the following partial result for a subsequence of natural numbers:

Theorem 2.5. For every natural number $n$, the set $\left\{f: \mathbb{R}^{2^{n}} \rightarrow \mathbb{R}^{2^{n}}: f\right.$ is linear and bijective $\}$ is $2^{n}$-lineable. In particular, the set of continuous injective functions $\mathbb{R}^{2^{n}} \rightarrow \mathbb{R}^{2^{n}}$ is $2^{n}$-lineable.

The proof of this result relies in the following proposition, which we believe has its own interest:

Proposition 2.6. There exist $2^{n}$ symmetries $\Phi_{1}^{(n)}=I, \Phi_{2}^{(n)}, \ldots, \Phi_{2^{n}}^{(n)}$ of the cube such that $\left\{\Phi_{1}^{(n)}(\mathbf{x}), \Phi_{2}^{(n)}(\mathbf{x}), \ldots, \Phi_{2^{n}}^{(n)}(\mathbf{x})\right\}$ is an orthogonal basis of $\mathbb{R}^{2^{n}}$, for any $\mathbf{x} \neq 0$.

Proof. We will proceed by induction on $n$. Assume first $n=1$. We may just choose

$$
\Phi_{2}^{(1)}=\left[\begin{array}{cc}
0 & -1 \\
-1 & 0
\end{array}\right],
$$

where we have identified $\Phi_{2}^{(1)}$ with the matrix defining a linear transformation. Assume next $n=2$. In this case we may consider, similarly, the transformations

$$
\Phi_{2}^{(2)}=\left[\begin{array}{cccc}
0 & 0 & 0 & 1 \\
0 & 0 & 1 & 0 \\
0 & -1 & 0 & 0 \\
-1 & 0 & 0 & 0
\end{array}\right], \Phi_{3}^{(2)}=\left[\begin{array}{cccc}
0 & 1 & 0 & 0 \\
-1 & 0 & 0 & 0 \\
0 & 0 & 0 & 1 \\
0 & 0 & -1 & 0
\end{array}\right], \Phi_{4}^{(2)}=\left[\begin{array}{cccc}
0 & 0 & 1 & 0 \\
0 & 0 & 0 & -1 \\
-1 & 0 & 0 & 0 \\
0 & 1 & 0 & 0
\end{array}\right]
$$


If $\mathbf{x}=(a, b, c, d)$, then

$$
\left(\Phi_{1}^{(2)}(\mathbf{x}), \Phi_{2}^{(2)}(\mathbf{x}), \Phi_{3}^{(2)}(\mathbf{x}), \Phi_{4}^{(2)}(\mathbf{x})\right)=\left[\begin{array}{cccc}
a & b & c & d \\
b & -a & d & -c \\
c & -d & -a & b \\
d & c & -b & -a
\end{array}\right]
$$

and it is straightforward to see that the above is an orthogonal set.

Assume finally that for $n \geq 2$ the result holds true and let us prove the result for $n+1$.

Denote, for $x=\left(x_{1}, \ldots, x_{2^{n+1}}\right), x^{\prime}=\left(x_{1}, \ldots x_{2^{n}}\right), x^{\prime \prime}=\left(x_{2^{n}+1}, \ldots x_{2^{n+1}}\right)$ and define the operators

$$
\begin{aligned}
\Phi_{i}^{(n+1)}\left(\begin{array}{c}
x^{\prime} \\
x^{\prime \prime}
\end{array}\right) & =\left(\begin{array}{c}
\Phi_{i}^{(n)}\left(x^{\prime}\right) \\
\Phi_{i}^{(n)}\left(x^{\prime \prime}\right)
\end{array}\right), \quad \text { if } 1 \leq i \leq 2^{n-1}, \\
\Phi_{2^{n}+i}^{(n+1)}\left(\begin{array}{c}
x^{\prime} \\
x^{\prime \prime}
\end{array}\right) & =\left(\begin{array}{c}
\Phi_{i}^{(n)}\left(x^{\prime \prime}\right) \\
-\Phi_{i}^{(n)}\left(x^{\prime}\right)
\end{array}\right), \\
\Phi_{i}^{(n+1)}\left(\begin{array}{c}
x^{\prime} \\
x^{\prime \prime}
\end{array}\right) & =\left(\begin{array}{c}
(n)\left(x^{\prime}\right) \\
-\Phi_{i}^{(n)}\left(x^{\prime \prime}\right)
\end{array}\right) \quad \text { if } 2^{n-1}+1 \leq i \leq 2^{n}, \\
\Phi_{2^{n}+i}^{(n+1)}\left(\begin{array}{c}
x^{\prime} \\
x^{\prime \prime}
\end{array}\right) & =\left(\begin{array}{c}
\Phi_{i}^{(n)}\left(x^{\prime \prime}\right) \\
\Phi_{i}^{(n)}\left(x^{\prime}\right)
\end{array}\right),
\end{aligned}
$$

Let us show that $\left\{\Phi_{1}^{(n+1)}(x), \ldots, \Phi_{2^{n+1}}^{(n+1)}(x)\right\}$ constitutes an orthogonal system. For that, let us focus on the rows $j$ and $k$, and let us denote

$$
\Phi_{i}^{(n+1)}(x)=\left({ }^{l} \Phi_{i}^{(n+1)}(x)\right)_{l=1}^{2^{n+1}},
$$

that is, ${ }^{l} \Phi_{i}^{(n+1)}$ is the $l$-th component of $\Phi_{i}^{(n+1)}$ for $1 \leq l \leq 2^{2^{n+1}}$. If first we assume $1 \leq j, k \leq 2^{n}$, then we need to compare the vectors

$$
\begin{aligned}
& \left({ }^{j} \Phi_{1}^{(n)}(x), \ldots,{ }^{j} \Phi_{2^{n-1}}^{n}(x),{ }^{j} \Phi_{2^{n-1}+1}^{(n)}(x), \ldots,\right. \\
& \left.\quad{ }^{j} \Phi_{2^{n}}^{(n)}(x),{ }^{j} \Phi_{1}^{(n)}(y), \ldots,{ }^{j} \Phi_{2^{n-1}}^{n}(y),{ }^{j} \Phi_{2^{n-1}+1}^{(n)}(y), \ldots,{ }^{j} \Phi_{2^{n}}^{(n)}(y)\right) \quad \text { and } \\
& \left({ }^{k} \Phi_{1}^{(n)}(x), \ldots,{ }^{k} \Phi_{2^{n-1}}^{n}(x),{ }^{k} \Phi_{2^{n-1}+1}^{(n)}(x), \ldots,\right. \\
& \left.\quad{ }^{k} \Phi_{2^{n}}^{(n)}(x),{ }^{k} \Phi_{1}^{(n)}(y), \ldots,{ }^{k} \Phi_{2^{n-1}}^{n}(y),{ }^{k} \Phi_{2^{n-1}+1}^{(n)}(y), \ldots,{ }^{k} \Phi_{2^{n}}^{(n)}(y)\right),
\end{aligned}
$$

which are orthogonal (by induction hypothesis).

If we assume next $2^{n}+1 \leq j, k \leq 2^{n+1}$, then by the same argument we also have orthogonality. Assume finally $1 \leq j \leq 2^{n}$ and $2^{n}+1 \leq k \leq 2^{n+1}$. Then, we need to compare the vectors

$$
\begin{aligned}
& \left({ }^{j} \Phi_{1}^{(n)}(x), \ldots,{ }^{j} \Phi_{2^{n-1}}^{n}(x),{ }^{j} \Phi_{2^{n-1}+1}^{(n)}(x), \ldots,\right. \\
& \left.\quad{ }^{j} \Phi_{2^{n}}^{(n)}(x),{ }^{j} \Phi_{1}^{(n)}(y), \ldots,{ }^{j} \Phi_{2^{n-1}}^{n}(y),{ }^{j} \Phi_{2^{n-1}+1}^{(n)}(y), \ldots,{ }^{j} \Phi_{2^{n}}^{(n)}(y)\right) \quad \text { and } \\
& \left({ }^{k} \Phi_{1}^{(n)}(y), \ldots,{ }^{k} \Phi_{2^{n-1}}^{n}(y),-{ }^{k} \Phi_{2^{n-1}+1}^{(n)}(y), \ldots,\right. \\
& \left.\quad-{ }^{k} \Phi_{2^{n}}^{(n)}(y),-{ }^{k} \Phi_{1}^{(n)}(x), \ldots,-{ }^{k} \Phi_{2^{n-1}}^{n}(x),{ }^{k} \Phi_{2^{n-1}+1}^{(n)}(x), \ldots,{ }^{k} \Phi_{2^{n}}^{(n)}(x)\right),
\end{aligned}
$$


and one can directly compute the inner product and show that is zero. This makes the induction step complete.

Proof of theorem 2.5. Consider the functions $\Phi_{i}=\Phi_{i}^{2^{n}}\left(1 \leq i \leq 2^{n}\right)$ given by Proposition 2.6. Let

$$
g(\mathbf{x}):=\sum_{i=1}^{2^{n}} \lambda_{i} \Phi_{i}(\mathbf{x})
$$

We will have the result if we succeed in proving that $g$ is injective for any nontrivial choice of $\lambda_{i} \in \mathbb{R}$. Since $g$ is linear, we must show that $g^{-1}(\{0\})=\{0\}$. To this aim, let $\mathbf{x} \in g^{-1}(\{0\})$. Then

$$
\sum_{i=1}^{2^{n}} \lambda_{i} \Phi_{i}(\mathbf{x})=0 .
$$

If we write $\Phi_{i}=\left(\Phi_{i}^{1}, \Phi_{i}^{2}, \ldots \Phi_{i}^{2^{n}}\right)$, then for each $1 \leq j \leq 2^{n}$ we obtain

$$
\sum_{i=1}^{2^{n}} \lambda_{i} \Phi_{i}^{j}(\mathbf{x})=0
$$

We can write this equality as the homogeneous system

$$
\left[\begin{array}{cccc}
\Phi_{1}^{1}(\mathbf{x}) & \Phi_{2}^{1}(\mathbf{x}) & \ldots & \Phi_{2^{n}}^{1}(\mathbf{x}) \\
\Phi_{1}^{2}(\mathbf{x}) & \Phi_{2}^{2}(\mathbf{x}) & \ldots & \Phi_{2^{n}}^{2}(\mathbf{x}) \\
\vdots & \vdots & \ddots & \vdots \\
\Phi_{1}^{2^{n}}(\mathbf{x}) & \Phi_{2}^{2^{n}}(\mathbf{x}) & \ldots & \Phi_{2^{n}}^{2^{n}}(\mathbf{x})
\end{array}\right]\left[\begin{array}{c}
\lambda_{1} \\
\lambda_{2} \\
\vdots \\
\lambda_{2^{n}}
\end{array}\right]=\left[\begin{array}{c}
0 \\
0 \\
0 \\
\vdots \\
0
\end{array}\right]
$$

By Proposition 2.6, the matrix of this system is non-degenerate if $\mathbf{x}$ is no zero. But the system has the non-trivial solution $\left(\lambda_{1}, \ldots, \lambda_{2^{n}}\right)$. Then $\mathbf{x}=0$ as required.

Remark 2.7. The proof of Theorem 2.5 is greatly supported by Proposition 2.6, but in fact we do not require the strong condition given there: it will be enough if, given a natural number $m=2^{n}$, we can guarantee the existence of linear operators $A_{2}, \ldots, A_{m}: \mathbb{R}^{m} \rightarrow$ $\mathbb{R}^{m}$ such that, for every $x \in \mathbb{R}^{m} \backslash\{0\},\left\{x, A_{2} x, \ldots, A_{m} x\right\}$ is a linearly independent set. The last is somehow also a necessary condition for an arbitrary $m \in \mathbb{N}$. Indeed, it is easy to see that the family of bijective linear functions $\mathbb{R}^{m} \rightarrow \mathbb{R}^{m}$ is l-lineable if and only if there are bijective linear functions $f_{1}, \ldots, f_{l}: \mathbb{R}^{m} \rightarrow \mathbb{R}^{m}$ such that, for every $x \in \mathbb{R}^{m} \backslash\{0\}$, the set $\left\{f_{1}(x), \ldots, f_{l}(x)\right\}$ is a linearly independent system.

Note that letting $n=2$ in Theorem 2.5 we obtain that the family of bijective linear functions $\mathbb{R}^{4} \rightarrow \mathbb{R}^{4}$ is 4 -lineable. On the contrary, the corresponding assertion fails for $\mathbb{R}^{3}$ :

Corollary 2.8. The set $\left\{f: \mathbb{R}^{3} \rightarrow \mathbb{R}^{3}: f\right.$ is linear and bijective $\}$ is not 3-lineable. 
Proof. Let us proceed by way of contradiction. According to Remark 2.7, we can assume, without loss of generality, that we can find non-singular linear operators $A$ and $B$ on $\mathbb{R}^{3}$ so that, for every $v=(x, y, z) \neq 0, \operatorname{det}[v, A v, B v] \neq 0$, that is

$$
\begin{aligned}
0 \neq & x^{3} \operatorname{det}\left[e_{1}, A e_{1}, B e_{1}\right]+x^{2} y\left(\operatorname{det}\left[e_{1}, A e_{1}, B e_{2}\right]+\operatorname{det}\left[e_{1}, A e_{2}, B e_{1}\right]+\operatorname{det}\left[e_{2}, A e_{1}, B e_{1}\right]\right) \\
+ & x^{2} z\left(\operatorname{det}\left[e_{1}, A e_{1}, B e_{3}\right]+\operatorname{det}\left[e_{1}, A e_{3}, B e_{1}\right]+\operatorname{det}\left[e_{3}, A e_{1}, B e_{1}\right]\right) \\
& +y^{3} \operatorname{det}\left[e_{2}, A e_{2}, B e_{2}\right] \\
& +y^{2} x\left(\operatorname{det}\left[e_{2}, A e_{2}, B e_{1}\right]+\operatorname{det}\left[e_{2}, A e_{1}, B e_{2}\right]+\operatorname{det}\left[e_{1}, A e_{2}, B e_{2}\right]\right) \\
& +y^{2} z\left(\operatorname{det}\left[e_{2}, A e_{2}, B e_{3}\right]+\operatorname{det}\left[e_{2}, A e_{3}, B e_{2}\right]+\operatorname{det}\left[e_{3}, A e_{2}, B e_{2}\right]\right) \\
+ & z^{3} \operatorname{det}\left[e_{3}, A e_{3}, B 3\right] \\
& +z^{2} x\left(\operatorname{det}\left[e_{3}, A e_{3}, B e_{1}\right]+\operatorname{det}\left[e_{3}, A e_{1}, B e_{3}\right]+\operatorname{det}\left[e_{1}, A e_{3}, B e_{3}\right]\right) \\
& +z^{2} y\left(\operatorname{det}\left[e_{3}, A e_{3}, B e_{2}\right]+\operatorname{det}\left[e_{3}, A e_{2}, B e_{3}\right]+\operatorname{det}\left[e_{2}, A e_{3}, B e_{3}\right]\right) \\
+ & x y z\left(\operatorname{det}\left[e_{1}, A e_{2}, B e_{3}\right]+\operatorname{det}\left[e_{1}, A e_{3}, B e_{2}\right]+\operatorname{det}\left[e_{2}, A e_{1}, B e_{3}\right]\right. \\
& \left.+\operatorname{det}\left[e_{2}, A e_{3}, B e_{1}\right]+\operatorname{det}\left[e_{3}, A e_{1}, B e_{2}\right]+\operatorname{det}\left[e_{3}, A e_{2}, B e_{1}\right]\right),
\end{aligned}
$$

where $e_{1}=(1,0,0), e_{2}=(0,1,0)$ and $e_{3}=(0,0,1)$.

We are dealing then with a homogeneous polynomial, $P(v=(x, y, z))=$ $\operatorname{det}[v, A v, B v]$, of degree 3 , since in particular we have

$$
\operatorname{det}\left[e_{1}, A e_{1}, B e_{1}\right] \cdot \operatorname{det}\left[e_{2}, A e_{2}, B e_{2}\right] \cdot \operatorname{det}\left[e_{3}, A e_{3}, B_{3}\right] \neq 0 .
$$

If we focus our attention on the points of the form $(x, 1,0)$, we have that $P(x, 1,0)$ is a polynomial of one variable of degree 3 , so in particular vanishes at one point $x_{0} \in \mathbb{R}$. Therefore, $\left\{\left(x_{0}, 1,0\right), A\left(x_{0}, 1,0\right), B\left(x_{0}, 1,0\right)\right\}$ cannot be a linearly independent set. This is the desired contradiction.

Remark 2.9. An analogous proof to the one in Corollary 2.8 would show that, for every natural number $k$, the set $\left\{f: \mathbb{R}^{2 k+1} \rightarrow \mathbb{R}^{2 k+1}: f\right.$ is linear and bijective $\}$ is not $(2 k+1)$-lineable.

Yet, we can have the partial positive result (from Theorem 2.5):

Corollary 2.10. For $n \in \mathbb{N}$, write $n=2^{k} m$, where $2 \nmid m$. Then, the set

$$
\left\{f: \mathbb{R}^{n} \rightarrow \mathbb{R}^{n} \text { such that } f \text { is linear and bijective }\right\}
$$

is $2^{k}$-lineable. In particular, the set

$$
\left\{f: \mathbb{R}^{n} \rightarrow \mathbb{R}^{n} \text { such that } f \text { is continuous and injective }\right\}
$$

is $2^{k}$-lineable.

Proof. Let $f_{1}, \ldots, f_{2^{k}}$ be a basis giving the $2^{k}$-lineability of the set

$$
\left\{f: \mathbb{R}^{2^{k}} \rightarrow \mathbb{R}^{2^{k}} \text { such that } f \text { is linear and bijective }\right\} .
$$

Define $\bar{f}_{i}: \mathbb{R}^{n} \rightarrow \mathbb{R}^{n}$, for $1 \leq i \leq 2^{k}$, as follows:

$$
\bar{f}_{i}\left(x_{1}, \ldots, x_{n}\right)=\left(f_{i}\left(x_{1}, \ldots, x_{2^{k}}\right), f_{i}\left(x_{2^{k}+1}, \ldots, x_{2 \cdot 2^{k}}\right), \ldots, f_{i}\left(x_{(m-1) 2^{k}+1}, \ldots, x_{m 2^{k}}\right)\right) .
$$

Then, the functions $\bar{f}_{1}, \ldots, \bar{f}_{2^{k}}$ give the desired result. 
All these considerations motivate the following open problem:

Question 2.11. What can we say about Theorem 2.5 for any dimension? Specifically, we are interested in the case when the dimension is even. Also, and taking into account what we have shown in Corollary 2.8, is $\left\{f: \mathbb{R}^{3} \rightarrow \mathbb{R}^{3}\right.$ such that $f$ is injective and continuous\} (not) 3-lineable?

Continuing with the consequences of Theorem 2.5, we have the following result that deals with infinite dimensions:

Theorem 2.12. Let $X$ be a real Banach space over $\mathbb{R}$ with Schauder basis $\left\{x_{j}\right\}_{j=0}^{\infty}$ and let $L(X)$ denote, as usual, the vector space of all operators (that is, the vector space of all continuous linear self-mappings). We have that $\{T \in L(X)$ such that $T$ is injective $\}$ is lineable.

Proof. Assume that the Schauder basis of the Banach space $X,\left\{x_{j}\right\}_{j=0}^{\infty}$, is normalized and let $V_{n}$ be a vector space provided by the $2^{n}$-lineability (see Theorem 2.5) of the set

$$
\left\{f: \mathbb{R}^{2^{n}} \rightarrow \mathbb{R}^{2^{n}} \text { such that } f \text { is linear and injective }\right\} .
$$

Let $\left\{f_{i}^{(n)}\right\}_{i=1}^{2^{n}}$ be a basis of $V_{n}$.

Arrange then the elements of $\cup_{n \in \mathbb{N}} V_{n}$ in a matrix whose $k$ th column consists of the functions $f_{1}^{(k-1)}, \ldots, f_{2^{k-1}}^{(k-1)}$ for the first $2^{k-1}$ entries, and $f_{2^{k-1}}^{(k-1)}$ for the other entries. That is, we create the following matrix:

$$
\left[\begin{array}{ccccccc}
f_{1}^{(0)} & f_{1}^{(1)} & f_{1}^{(2)} & f_{1}^{(3)} & f_{1}^{(4)} & f_{1}^{(5)} & \ldots \\
f_{1}^{(0)} & f_{2}^{(1)} & f_{2}^{(2)} & f_{2}^{(3)} & f_{2}^{(4)} & f_{2}^{(5)} & \ldots \\
f_{1}^{(0)} & f_{2}^{(1)} & f_{3}^{(2)} & f_{3}^{(3)} & f_{3}^{(4)} & f_{3}^{(5)} & \ldots \\
f_{1}^{(0)} & f_{2}^{(1)} & f_{4}^{(2)} & f_{4}^{(3)} & f_{4}^{(4)} & f_{4}^{(5)} & \ldots \\
f_{1}^{(0)} & f_{2}^{(1)} & f_{4}^{(2)} & f_{5}^{(3)} & f_{5}^{(4)} & f_{5}^{(5)} & \ldots \\
f_{1}^{(0)} & f_{2}^{(1)} & f_{4}^{(2)} & f_{6}^{(3)} & f_{6}^{(4)} & f_{6}^{(5)} & \ldots \\
f_{1}^{(0)} & f_{2}^{(1)} & f_{4}^{(2)} & f_{7}^{(3)} & f_{7}^{(4)} & f_{7}^{(5)} & \ldots \\
\vdots & \vdots & \vdots & \vdots & \vdots & \vdots & \ddots
\end{array}\right]
$$

Let $K \geq 1$ be the basis constant associated to the Schauder basis $\left\{x_{j}\right\}_{j=0}^{\infty}$. Then, if $x=\sum_{j=0}^{\infty} a_{j} x_{j}$ is the unique representation of $x \in X$ given by the Schauder basis $\left\{x_{j}\right\}_{j=0}^{\infty}$, we have that $\left|a_{j}\right|=\left|a_{j}\right|\left\|x_{j}\right\| \leq 2 K\|x\|$ for all $j \geq 0$ (see e.g. [5, Chap. 2]).

Consider then the subset $A=\left\{\mathbf{F}_{i}\right\}_{i \in \mathbf{N}} \subset \prod_{n=0}^{\infty} V_{n}$ whose elements are the rows of the previous matrix. Notice that each $\mathbf{F}_{i}$ has the form $\mathbf{F}_{i}=\left(f_{i_{0}}^{(0)}, f_{i_{1}}^{(1)}, f_{i_{2}}^{(2)}, \ldots\right)$. Let $f_{i}^{(j)}\left(a_{0}, a_{1}, \ldots, a_{2^{j}-1}\right)=\left(b_{j, 0}, \ldots, b_{j, 2^{j}-1}\right) \in \mathbf{R}^{2^{j}}$ and define $c_{n}:=\frac{b_{j, n-2^{j}}}{5^{j}}$ whenever $2^{j} \leq n<2^{j+1}(j=0,1,2, \ldots)$. Next, define $T\left(\mathbf{F}_{i}\right): X \rightarrow X$ by

$$
T\left(\mathbf{F}_{i}\right)(x)=\sum_{n=1}^{\infty} c_{n} x_{n}
$$


Now, by the selection of the functions $f_{i}^{(j)}\left(1 \leq i \leq 2^{j}\right)$ (see Theorem 2.5 and Proposition 2.6) we infer that the absolute value of each of the $2^{j}$ coordinates $b_{j, k}$ of the vector $f_{i}^{(j)}\left(a_{0}, a_{1}, \ldots, a_{2^{j}-1}\right)$ is not bigger than $\left|a_{0}\right|+\left|a_{1}\right|+\cdots+\left|a_{2^{j}-1}\right|$, and so not bigger than $2^{j} \cdot 2 K\|x\|$. Hence

$$
\begin{aligned}
\sum_{n=1}^{\infty}\left\|c_{n} x_{n}\right\| & =\sum_{j=0}^{\infty} \sum_{n=2^{j}}^{2^{j+1}-1}\left|c_{n}\right|\left\|x_{n}\right\|=\sum_{j=0}^{\infty} \sum_{n=2^{j}}^{2^{j+1}-1}\left|\frac{b_{j, n-2^{j}}}{5^{j}}\right| \\
& \leq \sum_{j=0}^{\infty} \frac{2^{j} \cdot 2^{j} \cdot 2 K\|x\|}{5^{j}}=10 K\|x\|<\infty,
\end{aligned}
$$

which together with the completeness of $X$ implies that $\sum_{n=1}^{\infty} c_{n} x_{n}$ converges in $X$. In other words, $T\left(\mathbf{F}_{i}\right)$ is well defined. Obviously, it is linear. Now, a simple application of the closed graph theorem (see e.g. [15, Chap. 2]) implies its continuity. Consequently, each $T\left(\mathbf{F}_{i}\right) \in L(X)$.

To conclude the result we shall show that every nontrivial linear combination of the set $\left\{T\left(\mathbf{F}_{i}\right): i \in \mathbb{N}\right\}$ yields an injective function. Thanks to linearity, this is equivalent to prove that the kernel of each such combination collapses to $\{0\}$.

Indeed, let $f(x)=\sum_{i=1}^{n} \lambda_{i} T\left(\mathbf{F}_{i}\right)(x)$ and assume $f\left(\sum_{j=0}^{\infty} a_{j} x_{j}\right)=0$. Let $k \geq 0$ so that $2^{k}>n$. Then, if we focus on the coordinates between $2^{k}$ and $2^{k+1}-1$ we would have

$$
\begin{aligned}
\frac{1}{5^{k}}\left(\lambda_{1} f_{1}^{(k)}\left(a_{0}, a_{1}, \ldots, a_{2^{k}-1}\right)+\lambda_{2} f_{2}^{(k)}\left(a_{0}, a_{1}, \ldots, a_{2^{k}-1}\right)\right. & +\ldots \\
& \left.+\lambda_{n} f_{n}^{(k)}\left(a_{0}, a_{1}, \ldots, a_{2^{k}-1}\right)\right)=0
\end{aligned}
$$

If $\left\{\lambda_{i}\right\}_{i=1}^{n}$ does not describe a trivial linear combination, we must conclude $a_{i}=0$ for every $0 \leq i \leq 2^{k}-1$, due to the injectivity of $\lambda_{1} f_{1}^{(k)}+\ldots+\lambda_{n} f_{n}^{(k)}$. Let $k \rightarrow \infty$ to obtain the result.

We remark that the previous result cannot be improved for a general infinite dimensional topological space, in the following sense:

Theorem 2.13. The set $\left\{f: c_{00} \rightarrow c_{00}\right.$ such that $f$ is injective $\}$ is not $\kappa$-lineable, for any cardinal number $\kappa>\aleph_{0}$.

Proof. Assume that we have a linearly independent set $\left\{f_{\alpha}\right\}_{\alpha \in \Gamma}$ of cardinality $\kappa>\aleph_{0}$. Denote $f_{\alpha}=\left(f_{\alpha}^{(1)}, f_{\alpha}^{(2)}, f_{\alpha}^{(3)}, \ldots\right)$ and fix $x$, an element of $c_{00}$. Then, there must exist a natural number $n_{0}$ so that, if $\Gamma_{n_{0}}:=\left\{\alpha \in \Gamma: f_{\alpha}^{(n)}(x)=0\right.$ for every $\left.n>n_{0}\right\}$, then $\# \Gamma_{n_{0}}>\aleph_{0}$.

Let $\left(y_{1}, \ldots, y_{n_{0}}\right) \neq\left(x_{1}, \ldots, x_{n_{0}}\right)$. By the same reason as before, there must exist a natural number $m_{0}$ such that, if $\Gamma^{m_{0}}=\left\{\alpha \in \Gamma_{n_{0}}: f_{\alpha}^{(m)}\left(y_{1}, \ldots, y_{n_{0}}, x_{n_{0}+1}, \ldots\right)=0\right.$ for every $\left.m>m_{0}\right\}$, then $\# \Gamma^{m_{0}}>\aleph_{0}$. 
Take $k_{0}=\max \left\{n_{0}, m_{0}\right\}$ and $\alpha_{1}, \ldots, \alpha_{k_{0}+1}$ elements of $\Gamma_{n_{0}}$ (if $k_{0}=n_{0}$ ) or $\Gamma^{m_{0}}$ (if $\left.k_{0}=m_{0}\right)$ and define, for every $1 \leq i \leq k_{0}+1$,

$$
\begin{gathered}
\tilde{f}_{\alpha_{i}}\left(z_{1}, \ldots, z_{k_{0}}\right):=\left(f_{\alpha_{i}}^{(1)}\left(z_{1}, \ldots, z_{k_{0}}, x_{k_{0}+1}, \ldots\right), f_{\alpha_{i}}^{(2)}\left(z_{1}, \ldots, z_{k_{0}}, x_{k_{0}+1}, \ldots\right), \ldots\right. \\
\left.\ldots, f_{\alpha_{i}}^{\left(k_{0}\right)}\left(z_{1}, \ldots, z_{k_{0}}, x_{k_{0}+1}, \ldots\right), 0,0, \ldots\right) .
\end{gathered}
$$

Then, $\tilde{f}_{\alpha_{i}}: \mathbb{R}^{k_{0}} \rightarrow \mathbb{R}^{k_{0}}$ and therefore there must exist nonzero real numbers $b_{1}, \ldots, b_{k_{0}+1}$ so that

$$
\sum_{i=1}^{k_{0}+1} b_{i} \tilde{f}_{\alpha_{i}}\left(x_{1}, \ldots, x_{k_{0}}\right)=\sum_{i=1}^{k_{0}+1} b_{i} \tilde{f}_{\alpha_{i}}\left(y_{1}, \ldots, y_{k_{0}}\right)
$$

Hence,

$$
\begin{aligned}
& \sum_{i=1}^{k_{0}+1} b_{i} f_{\alpha_{i}}\left(x_{1}, x_{2}, \ldots, x_{k_{0}}, x_{k_{0}+1}, \ldots\right) \\
= & \left(\sum_{i=1}^{k_{0}+1} b_{i} f_{\alpha_{i}}^{(1)}\left(x_{1}, \ldots, x_{k_{0}}\right), \sum_{i=1}^{k_{0}+1} b_{i} f_{\alpha_{i}}^{(2)}\left(x_{1}, \ldots, x_{k_{0}}\right), \sum_{i=1}^{k_{0}+1} b_{i} f_{\alpha_{i}}^{\left(k_{0}\right)}\left(x_{1}, \ldots, x_{k_{0}}\right), 0,0, \ldots,\right) \\
= & \left(\sum_{i=1}^{k_{0}+1} b_{i} f_{\alpha_{i}}^{(1)}\left(y_{1}, \ldots, y_{k_{0}}\right), \sum_{i=1}^{k_{0}+1} b_{i} f_{\alpha_{i}}^{(2)}\left(y_{1}, \ldots, y_{k_{0}}\right), \sum_{i=1}^{k_{0}+1} b_{i} f_{\alpha_{i}}^{\left(k_{0}\right)}\left(y_{1}, \ldots, y_{k_{0}}\right), 0,0, \ldots\right) \\
= & \sum_{i=1}^{k_{0}+1} b_{i} f_{\alpha_{i}}\left(y_{1}, y_{2}, \ldots, y_{k_{0}}, x_{k_{0}+1}, \ldots\right),
\end{aligned}
$$

so there exists a non trivial linear combination of the functions $f_{\alpha_{1}}, \ldots, f_{\alpha_{k_{0}+1}}$ which is not injective.

Question 2.14. We would like to remark that $c_{00}$ is not a Banach space. Therefore we have the following open question for this problem: Can we improve Theorem 2.12 for concrete spaces, like $c_{0}$ ? Also, can we prove that the set there considered cannot be further lineable in general? Concerning the vector space $c_{00}$, can we have an analogous theorem to Theorem 2.12 for this specific case?

\section{Some remarks on nowhere differentiability}

In [13], the authors used the functions $K_{a, b}$ defined by Knopp in some results in their article. By $\mathbb{Z}$ we denote, as usual, the set of all integer numbers.

Definition 3.1. Let $0<a<1$ and $b>1$ so that $1 / a>a b>1$. Denote $f_{b, k}(x)=$ $\operatorname{dist}\left(b^{k} x, \mathbb{Z}\right)$ and define the Knopp's function as

$$
K_{a, b}(x)=\sum_{k \geq 0} a^{k} f_{b, k}(x)
$$

This function is a continuous nowhere differentiable function, but convolving it with a function of the same kind gives differentiability at every point. 
Theorem 3.2. Let $K_{a}=K_{a, 9}$ for $\frac{1}{9}<a<\frac{1}{3}$. Then $K_{a_{1}} *_{V} K_{a_{2}}$ is differentiable everywhere for all $a_{1}, a_{2} \in\left(\frac{1}{9}, \frac{1}{3}\right)$.

In order to prove the above theorem, we first recall the following result, which follows from the well-known Weierstrass M-test:

Proposition 3.3. Let $\left(f_{n}\right)_{n=0}^{\infty}$ be a sequence of differentiable functions on an interval I of $\mathbb{R}$ and let $\left(a_{n}\right)_{n=0}^{\infty}$ be a sequence of numbers such that $\sum_{n=0}^{\infty}\left|a_{n}\right|<\infty$. Assume that $\sup _{n \geq 0} \sup _{x \in I}\left|f^{\prime}(x)\right|<\infty$ for all $n \geq 0$ and that $\sum_{n=0}^{\infty} a_{n} f_{n}(x)$ converges for at least one $x \in I$. Then $\sum_{n=0}^{\infty} a_{n} f_{n}$ converges uniformly on I to a differentiable function $f$ such that $f^{\prime}=\sum_{n=0}^{\infty} a_{n} f_{n}^{\prime}$.

Proof of Theorem 3.2. Denote $f_{k}=f_{9, k}$ (see Definition 3.1) and notice

$$
\begin{aligned}
K_{a_{1}} * V & K_{a_{2}}(x) \\
& =\int_{0}^{x} K_{a_{1}}(\tau) K_{a_{2}}(x-\tau) d \tau=\int_{0}^{x}\left(\sum_{k=0}^{\infty} a_{1}^{k} f_{k}(\tau)\right)\left(\sum_{j=0}^{\infty} a_{2}^{j} f_{j}(x-\tau)\right) d \tau \\
& =\sum_{k=0}^{\infty} \sum_{j=0}^{k} \int_{0}^{x} a_{1}^{k} \operatorname{dist}\left(9^{k} \tau, \mathbb{Z}\right) a_{2}^{k-j} \operatorname{dist}\left(9^{k-j}(x-\tau), \mathbb{Z}\right) d \tau \\
& =\sum_{k=0}^{\infty}\left(\frac{9 a_{1} a_{2}+1}{2}\right)^{k}\left(\frac{2}{9 a_{1} a_{2}+1}\right)^{k} \sum_{j=0}^{k} a_{1}^{k} a_{2}^{k-j} \int_{0}^{x} \operatorname{dist}\left(9^{k} \tau\right) \operatorname{dist}\left(9^{k-j}(x-\tau)\right) d \tau \\
& =\sum_{k=0}^{\infty}\left(\frac{9 a_{1} a_{2}+1}{2}\right)^{k} g_{k}(x),
\end{aligned}
$$

with

$$
g_{k}(x)=\left(\frac{2}{9 a_{1} a_{2}+1}\right)^{k} \sum_{j=0}^{k} a_{1}^{k} a_{2} k-j \int_{0}^{x} \operatorname{dist}\left(9^{k} \tau\right) \operatorname{dist}\left(9^{k-j}(x-\tau)\right) d \tau,
$$

where we have been able to interchange sum with integral sign using the fact that $0<a_{i}<1$, and therefore both infinite sums are convergent.

Each of the functions $g_{k}$ is differentiable, with

$$
g_{k}^{\prime}(x)=\left(\frac{2}{9 a_{1} a_{2}+1}\right)^{k} \sum_{j=0}^{k} a_{1}^{k} a_{2}^{k-j} \int_{0}^{x} \operatorname{dist}\left(9^{k} \tau\right) 9^{k-j} \operatorname{dist}^{\prime}\left(9^{k-j}(x-\tau)\right) d \tau,
$$

and hence,

$$
\left|g_{k}^{\prime}(x)\right| \leq\left(\frac{18 a_{1} a_{2}}{9 a_{1} a_{2}+1}\right)^{k} \sum_{j=0}^{k}\left(\frac{1}{9 a_{2}}\right)^{j} \frac{M}{2} \leq \frac{9 M a_{2}}{2\left(1-9 a_{2}\right)}
$$

In conclusion, $\left|g_{k}^{\prime}(x)\right| \leq \frac{9 M a_{2}}{2\left(1-9 a_{2}\right)}$, for all $x$ in $[0, M]$ and all $k$. Applying Proposition 3.3 it follows that $K_{a_{1}} *_{V} K_{a_{2}}$ is differentiable. 
Corollary 3.4. The family $\left\{K_{a}: \frac{1}{9}<a<\frac{1}{3}\right\}$ is a linearly independent set which spans a vector space of functions which, when convolving among themselves, give a differentiable function.

Proof. We only need to prove the linearly independence, just taking into account the linearity of the convolution and the differentiability.

Indeed, let $\lambda_{1}, \ldots, \lambda_{n}$ in $\mathbb{R}$ and $\frac{1}{9}<a_{1}, \ldots, a_{n}<\frac{1}{3}$, and consider

$$
f(x)=\sum_{i=1}^{n} \lambda_{i} K_{a_{i}}(x)
$$

Then, assuming $f \equiv 0$ and evaluating at $\frac{1}{9}, \frac{1}{9^{2}}, \ldots, \frac{1}{9^{n}}$ would give

$$
\sum_{i=1}^{n} \lambda_{i}=0, \sum_{i=1}^{n} \lambda_{i} a_{i}=0, \sum_{i=1}^{n} \lambda_{i} a_{i}^{2}=0, \ldots, \sum_{i=1}^{n} \lambda_{i} a_{i}^{n}=0,
$$

leading us to a Van der Monde determinant, which is nonzero because the $a_{i}{ }^{\prime} \mathrm{s}$ are pairwise different. This allows us to conclude that $\lambda_{i}=0$ for every $i$, as desired.

Acknowledgements. The authors express their gratitude to the Referee and Prof. Richard Aron, whose thorough analysis and insightful remarks improved the text. The authors would also like to thank Prof. Sergii Myroshnychenko for pointing out the result gathered here in the form of Proposition 2.6, which was crucial for Section 2. 


\section{References}

[1] N. Albuquerque, Maximal lineability of the set of continuous surjections, Bull. Belg. Math. Soc. Simon Stevin 21 (2014), no. 1, 83-87.

[2] R. M. Aron, L. Bernal-González, D. Pellegrino, and J. B. Seoane-Sepúlveda, Lineability: The search for linearity in Mathematics, Monographs and Research Notes in Mathematics, Chapman \& Hall/CRC, Boca Raton, FL, 2016, ISBN 978-1-48-229909-0.

[3] R. M. Aron, J. A. Conejero, A. Peris, and J. B. Seoane-Sepúlveda, Uncountably generated algebras of everywhere surjective functions, Bull. Belg. Math. Soc. Simon Stevin 17 (2010), no. 3, 571-575.

[4] R. M. Aron, V. I. Gurariy, and J. B. Seoane-Sepúlveda, Lineability and spaceability of sets of functions on $\mathbb{R}$, Proc. Amer. Math. Soc. 133 (2005), no. 3, 795-803 (electronic), DOI 10.1090/S0002-9939-04-07533-1.

[5] B. Beauzamy, Introduction to Banach spaces and their geometry, Mathematical Studies, 68, North Holland, Amsterdam, 1982.

[6] L. Bernal-González, D. Pellegrino, and J. B. Seoane-Sepúlveda, Linear subsets of nonlinear sets in topological vector spaces, Bull. Amer. Math. Soc. (N.S.) 51 (2014), no. 1, 71-130, DOI 10.1090/S0273-0979-2013-01421-6.

[7] P. H. Enflo, V. I. Gurariy, and J. B. Seoane-Sepúlveda, Some results and open questions on spaceability in function spaces, Trans. Amer. Math. Soc. 366 (2014), no. 2, 611-625, DOI 10.1090/S0002-9947-2013-05747-9.

[8] V. P. Fonf, V. I. Gurariy, and M. I. Kadets, An infinite dimensional subspace of $C[0,1]$ consisting of nowhere differentiable functions, C. R. Acad. Bulgare Sci. 52 (1999), no. 11-12, 13-16.

[9] J. L. Gámez-Merino, G. A. Muñoz-Fernández, V. M. Sánchez, and J. B. Seoane-Sepúlveda, Sierpiński-Zygmund functions and other problems on lineability, Proc. Amer. Math. Soc. 138 (2010), no. 11, 3863-3876, DOI 10.1090/S00029939-2010-10420-3.

[10] J. L. Gámez, G. A. Muñoz-Fernández, and J. B. Seoane-Sepúlveda, Lineability and additivity in $\mathbb{R}^{\mathbb{R}}$, J. Math. Anal. Appl. 369 (2010), no. 1, 265-272, DOI 10.1016/j.jmaa.2010.03.036.

[11] F. J. García-Pacheco, M. Martín, and J. B. Seoane-Sepúlveda, Lineability, spaceability, and algebrability of certain subsets of function spaces, Taiwanese J. Math. 13 (2009), no. 4, 1257-1269.

[12] W. Goldbloom Bloch, Open discontinuous maps from $\mathbb{R}^{n}$ onto $\mathbb{R}^{n}$, Amer. Math. Monthly 122 (2015), no. 3, 268-271, DOI 10.4169/amer.math.monthly.122.03.268.

[13] P. Jiménez-Rodríguez, S. Maghsoudi, and G. A. Muñoz-Fernández, Convolution functions that are nowhere differentiable, J. Math. Anal. Appl. 413 (2014), no. 2, 609-615, DOI 10.1016/j.jmaa.2013.12.008. 
[14] P. Jiménez-Rodríguez, G. A. Muñoz-Fernández, and J. B. Seoane-Sepúlveda, On Weierstrass' Monsters and lineability, Bull. Belg. Math. Soc. Simon Stevin 20 (2013), no. 4, 577-586.

[15] W. Rudin, Functional Analysis, McGraw-Hill Book Co., New York, 1991. 2nd edition.

[16] J. B. Seoane-Sepúlveda, Chaos and lineability of pathological phenomena in analysis, ProQuest LLC, Ann Arbor, MI, 2006. Thesis (Ph.D.)-Kent State University.

Department of Mathematical Sciences,

Mathematics and Computer Science Building,

Summit Street,

Kent OH 44242, USA.

email:pjimene1@kent.edu.

Department of Mathematics,

University of Zanjan,

Zanjan 45195-313, Iran.

email:s_maghsodi@znu.ac.ir

Departamento de Análisis Matemático,

Facultad de Ciencias Matemáticas,

Plaza de Ciencias 3,

Universidad Complutense de Madrid,

Madrid, 28040, Spain.

email:gustavo_fernandez@mat.ucm.es

Instituto de Ciencias Matemáticas (CSIC-UAM-UC3M-UCM)

C/ Nicolás Cabrera 13-15

Campus de Cantoblanco, 28049 Madrid, Spain

AND

Departamento de Análisis Matemático,

Facultad de Ciencias Matemáticas,

Plaza de Ciencias 3,

Universidad Complutense de Madrid,

28040 Madrid, Spain.

email:jseoane@ucm.es 